\title{
OS ASPECTOS TEÓRICOS E POLÍTICOS QUE FUNDAMENTAM A PRÁTICA DA ACUPUNTURA PELO EDUCADOR FÍSICO
}

\section{The political and theoretical aspects that ground its practice of acupuncture by the physical educator}

\author{
Ana Paula Serra De Araújo ${ }^{1}$; Rosane Sakuma $^{2}$ \\ ${ }^{1}$ Umuarama - Paraná - Brasil. \\ ${ }^{2}$ Marialva - Paraná - Brasil.
}

\begin{abstract}
Resumo: Nestes 11 anos de atividade reconhecida como de nível superior e da área de saúde (1998) a educação física vêm crescendo e ocupando novos espaços. Atualmente dentre as diversas especialidades do educador físico, temos a Acupuntura, que nos últimos anos tem gerado uma série de discussão e a criação de diversos projetos de lei que visam monopoliza-la como especialidade exclusiva dos profissionais médicos. O presente estudo teve por objetivo abordar os aspectos teóricos e políticos que fundamentam a prática da Acupuntura pelo educador físico mediante uma revisão de literatura. Concluindo-se que o educador físico como um profissional da área de saúde que estuda o corpo em diferentes dimensões, é apto e esta amparado legalmente para exercer a especialidade de Acupuntura desde que possua devida formação em Acupuntura conforme Resolução CONFEF $n^{\circ}$. 069/2003 que reconheceu a Acupuntura como um recurso cientifico e complementar dentro do campo de atuação do educador físico. Palavras Chave: Educador Físico; Acupuntura, Regulamentação profissional.
\end{abstract}

Abstract: In 11 years of activity structured as an undergraduate course of the health area (1998) physical education is growing and occupying new spaces. Currently, among the various specialties the physical educator may have, we have the Acupuncture, which, in recent years, has generated a series of discussion and the creation of several projects that aim to monopolize it as an exclusive specialty of medicine professionals. This study aimed to address the theoretical and political issues underlying the practice of Acupuncture by the physical educator through a literature review. It was concluded that the physical educators, as professionals in the health area that study the body in different dimensions, are capable and have legal support to perform the specialization of Acupuncture, since they are provided with appropriate training in Acupuncture, following the recommendations in CONFEF Resolution No. 069/2003 - which recognized Acupuncture as a complementary and scientific resources within the field of physical education.

Key Words: Physical Educator; Acupuncture; Professional Regulation.

Aceito em 22/07/2009 - Rev. Educ. Fís. 2009 - 32-39. Rio de Janeiro - RJ - Brasil

\section{INTRODUÇÃO}

No Brasil, a educação física é uma profissão antiga tendo sua institucionalização data na época do Brasil imperial em meados de 1822 a 1889, quando a atividade física ganhou espaço nas leis e decretos sobre educação física e desporto no país $(1,2)$.

Vista em meados do século passado como um ramo da área de educação e da medicina que visava cumprir a função de colaborador na construção de corpos saudáveis ${ }^{(3)}$.

No Brasil a educação física somente passou a ser consagrada como uma profissão de nível superior e da área de saúde em $1^{\circ}$ de setembro de 1998, após a criação da lei 9.696/98 que também deu origem aos conselhos federal e regionais de educação física (CONFEF/CREFs) no país e após a publicação da resolução 013/99 do CONFEF que ressaltou a importância dos serviços prestados pelos profissionais de educação física, para o bem estar de toda a população ${ }^{(3,4,5)}$.

Nos últimos 20 anos os conhecimentos em educação física avançaram, mas isto não significou uma melhora na atuação profissional do licenciado. Atualmente a intervenção profissional em educação física atravessa um período de transição onde há uma maior demanda por serviços e por profissionais mais qualificados bem como por cursos de graduação mais conscientes de suas responsabilidades ${ }^{(2)}$.

Embora atualmente a formação acadêmica em 
educação física vise formar profissionais para atuar em áreas específicas, atendendo a legislação e o mercado de trabalho. Os cursos de graduação em educação física têm hoje almejado cada vez mais formar profissionais aptos a planejar programas dentro de uma escola para a educação infantil, ensino fundamental, ensino médio como também por formar profissionais aptos para o atendimento à população em geral fora do contexto escolar (clínicas, clubes, spas, centros esportivos e etc.). Através de uma atuação profissional voltada para a manutenção e promoção da saúde ${ }^{(3)}$.

Isso tem feito com que a educação física nos dias atuais seja considerada uma prática social e não apenas uma prática pedagógica e esportiva, onde a formação permanente busca conjugar a experiência, a competência técnico-científica e comunicativa deste profissional a fim de contemplar uma adequação aos avanços tecnológicos, científicos e sociais ${ }^{(6)}$.

Cabendo ao profissional de educação física valorizar a busca de conhecimentos e lutar pelo desenvolvimento de sua profissão, levando seus serviços a toda população, sem qualquer tipo de descriminação, visando sempre o bem-estar do seu aluno/cliente ${ }^{(2,3)}$

Neste contexto de busca por novos conhecimentos e desenvolvimento da profissão desde a publicação da portaria $n^{\circ}$. 971, de 3 de maio de 2003 , art. $1^{\circ}$ e art. $2^{\circ}$ o CONFEF e CREF passaram a reconhecer a especialidade multiprofissional da Acupuntura como recurso científico complementar ao campo de atuação do profissional de educação física desde que este profissional, comprove formação especifica em nível de especialização e/ou pós graduação para o uso de tal técnica e desde que, este profissional respeite o disposto no seu respectivo código de ética profissional ${ }^{(7)}$.

O presente estudo tem por objetivo abordar os aspectos teóricos e políticos que fundamentam a prática da Acupuntura pelo educador físico mediante uma revisão de literatura.

\section{PROCEDIMENTOS METODOLÓGICOS}

O presente trabalho foi realizado mediante revisão de literatura, em livros, artigos e periódicos eletrônicos, que continham informações sobre: educação física, profissional de educação física, educação física no Brasil, reconhecimento da educação como profissão no Brasil, acupuntura, acupuntura no Brasil, reconhecimento da acupuntura como especialidade da área de saúde, projeto de lei ato médico, uso da acupuntura em educação física, acupuntura em atletas.

Os livros pesquisados foram publicados a partir de 1995 e os artigos, periódicos eletrônicos e trabalhos acadêmicos de conclusão de cursos acadêmicos (monografias, teses e dissertação) a partir de 1998. Foram excluídos todos os livros, artigos, periódicos eletrônicos e trabalhos acadêmicos de conclusão de cursos publicados antes do período acima referido e/ou os que não continham informações relevantes e/ou referentes ao tema abordado pelo presente trabalho.

Sendo os livros, artigos e trabalhos acadêmicos de conclusão de cursos pesquisados nas bibliotecas do Instituto Brasileiro de Therapias e Ensino (IBRATE), da Universidade Paranaense (UNIPAR) e na biblioteca do Centro Universitário de Maringá (CESUMAR). Já os artigos advindos de periódicos eletrônicos foram pesquisados nos sites: www.google.com, www.scielo.br e www. bireme.br.

\section{DISCUSSÃO}

Este estudo buscou abordar os principais aspectos teóricos e políticos que fundamentam a prática de acupuntura pelos profissionais de educação física. Devido à carência de estudos referentes a este assunto. Tomaram - se por base publicações relacionadas à regulamentação e história da educação física no Brasil, prática de acupuntura por profissionais da área de saúde médicos e não médicos, regulamentação e história da acupuntura como profissão no Brasil e sobre o uso da Acupuntura como coadjuvante no tratamento de atletas.

Conforme Almeida-Filho (8) que a pouco ou quase nada a se pesquisar em termos de jurisprudência sobre a prática da acupuntura enquanto profissão reconhecida e regulamentada no Brasil, o que se tem apenas são decisões recentes dos tribunais que se apresentamse posicionadas no sentido de não expurgar a especialidade de Acupunturista a uma ou outra classe profissional especifica.

Segundo Akiyama ${ }^{(2)}$ nos países ocidentais 
como o Brasil a prática da medicina complementar e/ou alternativa como a Acupuntura e seus vertentes dentre os quais temos a Auriculoterapia, Crânio Acupuntura, Quiroacupuntura, Fitoterapia entre outras técnicas vêm recebendo crescente atenção entre a classe médica devido ao aumento do seu uso pela população, que em certas regiões a sua prevalência de uso chega a atingir $40 \%$ da população em geral.

No Brasil, no entanto nos últimos anos vem havendo uma crescente discussão e disputa pela monopolização da prática de Acupuntura no país por apenas profissionais médicos. Tal tentativa de monopolização vem ocorrendo através da tentativa de se conseguir a aprovação do projeto de lei intitulado "Ato Médico". Neste projeto de lei conforme Almeida-Filho ${ }^{(8)}$ consta como ato médico:

"todo procedimento técnico-profissional praticado por médico habilitado e dirigido para: I-a promoção primária, definida como a promoção da saúde e a prevenção da ocorrência de enfermidades ou profilaxia; II - a prevenção secundária, definida como a prevenção da evolução das enfermidades ou execução de procedimentos diagnósticos ou terapêuticos; III - a prevenção terciária, definida como a prevenção da invalidez ou reabilitação dos enfermos. Parágrafo único. As atividades de prevenção de que trata este artigo, que envolvam procedimentos diagnósticos de enfermidades ou impliquem em indicação terapêutica, são atos privativos do profissional médico."

Conforme podemos observar no exposto acima este projeto de lei se aprovado implicará diretamente sobre o exercício da prática clínica de Acupuntura no Brasil, tornando a prática de Acupuntura exclusiva da classe médica no país. Embora o profissional habilitado e especializado em Acupuntura independe da sua área de formação, realiza diagnóstico, tratamento e prevenção de uma série de patologias e/ ou distúrbios baseados nos princípios teóricos da MTC (teoria do YinYang, do QI, dos cinco elementos e dos canais de energia) e não nos princípios da medicina ortodoxa.

Com a aprovação do projeto "Ato Médico" qualquer procedimento que envolva a execução de diagnóstico e prevenção de enfermidades será prática exclusiva do profissional médico. $\mathrm{O}$ que impediria então qualquer outro profissional de exercer a especialidade de Acupuntura. Porém até o presente momento como já foi dito nenhuma classe profissional no Brasil detém o monopólio sobre á prática de Acupuntura no país. Uma vez que no Brasil assim como em diversos países a Acupuntura pode ser praticada tanto por profissionais da área de saúde, como por qualquer outro indivíduo que possua formação especifica em Acupuntura em virtude da existência de cursos de formação técnica, aperfeiçoamento, especialização, pós - graduação e/ou graduação em Acupuntura, oferecidos por diversos institutos, faculdades e Universidades do país. E pelo fato de não existir hoje no país nenhuma lei federal que impressa o profissional devidamente habilitado/ especializado em Acupuntura de exerce - lá (10, 11, 12).

De acordo com um levantamento feito no ano de 2001, pela OMS em diversos países onde a Acupuntura é praticada, observou-se que em 50 países a Acupuntura é praticada legalmente por profissionais de diversas áreas desde que estes profissionais possuam formação/especialização em Acupuntura e que somente em 2 países (Arábia Saudita e Áustria) a prática de Acupuntura é restrita apenas a profissionais médicos ${ }^{(13)}$. Estes dados demonstram a supremacia do caráter multiprofissional da prática de Acupuntura no mundo e corroboram com a atual situação legal da prática de Acupuntura no Brasil.

Embora não haja uma previsão legal, sobre está questão "de quem pode ou não praticar a Acupuntura no Brasil", algumas decisões judiciais já vêm contemplando esta situação. Decisões estas que tem apoiado o caráter multiprofissional da prática da Acupuntura no país. Como no caso do reconhecimento da prática de Acupuntura como uma área de especialidade da educação física conforme o disposto na Resolução CONFEF $n^{\circ} .069 / 2003$ art. $1^{\circ}$ e $2^{\circ}(7)$ :

"Art. $1^{\circ}$ - Reconhecer a possibilidade de utilização da Técnica de Acupuntura, como recurso científico complementar, no desenvolvimento da intervenção do Profissional de Educação Física, devendo, portanto, respeitar a vida, a dignidade, a integridade e os direitos da pessoa humana, em particular, daqueles que são seus beneficiários.

Art. $2^{\circ}$ - O Profissional de Educação Física, componente da área da Saúde, dentro do universo de suas possibilidades de intervenção e 
ao exercer seu direito, poderá recorrer à Técnica de Acupuntura, desde que comprove formação especializada para seu uso, respeitando o disposto no Código de Ética do Profissional de Educação Física. ${ }^{(7)}$ "

A partir da publicação desta resolução, os profissionais de educação física especialistas em Acupuntura passaram a contam com o apoio das suas respectivas regionais (CREFS), que desde então vem regularizando todos os educadores físicos especialistas em Acupuntura junto ao CONFEF garantindo assim a legitimidade do exercício profissional da Acupuntura por estes profissionais ${ }^{(11,12)}$.

Esta regularização da Acupuntura como especialidade do profissional de educação física através da publicação da Resolução CONFEF $n^{\circ}$. 069/2003 veio a exemplo de iniciativas similares de diversos outros conselhos das áreas de saúde do Brasil, que desde o ano de 1985 vêm reconhecendo a Acupuntura como especialidade dos seus respectivos profissionais no país. Neste contexto temos que o primeiro conselho de saúde do Brasil a publicar resoluções que reconheciam a Acupuntura como uma terapia complementar a atuação profissional dos seus cadastrados foi o conselho de fisioterapia no ano de 1985, seguido pelo conselho de biomedicina em 1986, pelo de enfermagem e medicina em 1995, pelo de odontologia em 1999, pelo de farmácia em 2000, pelo de terapia ocupacional e fonoaudióloga em 2001, pelo de psicologia em 2002 e pelo de educação física em $2003^{(11,12,14)}$.

É válido ressaltar neste momento que o conselho federal de medicina (CFM), desde o início da busca pela regularização da prática clínica da Acupuntura no Brasil mostrou-se relutante em aceitar a Acupuntura como especialidade médica. Porém, com o Projeto de Lei PL 67/19956, o CFM retrocedeu em sua decisão reconhecendo a Acupuntura como uma especialidade médica em 1995. E desde o final do ano de 2001, com o apoio da Sociedade Medica Brasileira de Acupuntura (SMBA), o CFM vem movendo ações contra os demais conselhos das áreas de saúde do Brasil que possuíam resoluções regulamentadoras sobre a prática clínica de Acupuntura no Brasil por seus profissionais. Na tentativa assim de proibir que outros profissionais da área da saúde não médicos exercessam essa atividade, alegando que os demais profissionais da área de saúde não teriam capacidade para realizar o diagnóstico e aplicação clínica da Acupuntura (11, 12, 14, 15).

Ė inescapável neste momento lembrar que a Acupuntura é uma técnica da MTC que aborda holisticamente a saúde, não sendo usada apenas para promover o alívio de sintomas, mas também para auxiliar na regulação do organismo através da promoção de mecanismos homeostáticos ${ }^{(15)}$.

Seus princípios teóricos e práticos são totalmente distintos dos princípios teóricos e práticos da medicina ortodoxa/alopata. Uma vez que na MTC, de onde provém a técnica de Acupuntura e dezenas de outras técnicas como a Auriculoterapia, Moxabustão, Ventosaterapia, as quais têm por fundamentação estudar o homem e suas relações com a natureza, seus ciclos, movimentos e como estes se influenciam mutuamente ${ }^{(15,16)}$. Tem por compreensão que o universo e o homem interagem entre si e são nutridos por uma força (energia vital) conhecida como "Chi" e/ou "Qi" que assume diferentes padrões e/ou movimentos os quais se transmutam entre padrões de Yin (deficiência) e Yang (excesso) ao longo dos 12 canais de energia e/ ou meridianos de Acupuntura principais e pelos 8 canais de energia extraordinários que encontramse distribuídos por todo o corpo humano $(4,16,17)$.

Outro aspecto ainda muito importante sobre a prática clínica da Acupuntura e que esta não se baseia no modelo fisiológico e anatomofuncional impregnado no mundo ocidental, onde temos a medicina ortodoxa/alopata conforme pudemos observar ao longo do texto, não tendo, portanto nada haver com os métodos de diagnóstico e tratamento pregados pela medicina ortodoxa/ alopata (exames clínicos, laboratoriais e de imagem), mas sim se baseia na analise dos desequilíbrios energéticos conforme a teoria do Yin-Yang, análise do tipo de pulso, biótipo constitucional, sintomas físicos, personalidade etc $(4,10,15,16)$

Porém devidoà realização de inúmerosestudos neurofisiológicos realizados nas últimas décadas os quais vêm estabelecendo uma significativa aproximação entre a MTC e a medicina ortodoxa/ alopata, através da constatação de inúmeras interrelações existentes entre as duas medicinas nos últimos anos, além de virem comprovando os reais efeitos terapêuticos da utilização de 
tais técnicas no tratamento das mais variadas enfermidades. O que fez despertar nas últimas décadas o interesse de diferentes profissões da área de saúde pelo uso destas técnicas provindas da MTC nas suas respectivas áreas de atuação bem como também despertou o interesse da classe médica pela monopolização da especialização de Acupuntura que dentre as técnicas da MTC é amais difundida e pesquisada ${ }^{(10)}$.

Desta forma pode-se dizer que os médicos vêm nas técnicas da MTC, mais especificamente na Acupuntura uma forma de complementar a medicina ortodoxa/alopata. Já que existem várias evidencias científicas que comprovam a eficácia terapêutica desta técnica e a existência anatomofisiológica dos acupontos (Pontos de Acupuntura). Além é claro da existência de diversos estudos científicos desenvolvidos em diversas áreas das saúde dentre as quais se tem a ortopedia, reumatologia, neurológica entre outros, publicados em revistas cientificas nacionais e internacionais que por sua vez tem apontado vários indícios de êxito do uso da Acupuntura e seus vertentes durante o tratamento de pacientes com diversas doenças e/ou distúrbios de origem física e/ou psicológica ${ }^{(15,18)}$.

Já no campo de atuação do profissional de educação física a Acupuntura vêm sendo utilizadas ao longo dos últimos anos como um método capaz de potencializar o desempenho físico e o rendimento esportivo de atletas e de indivíduos comuns que praticam atividade física regularmente, bem como vem sendo utilizada como um método coadjuvante na recuperação e no tratamento de atletas lesionados ${ }^{(19,20,21)}$.

Embora sejam, poucos os estudos científicos publicados que falam sobre a utilização da Acupuntura recurso para a melhora da performance física de atletas, os poucos estudos publicados sobre este assunto conforme Luna \& Filho ${ }^{(20)}$ são contraditórios. Além é claro do fato de que a comunidade cientifica tem dado pouca atenção para a realização de estudo que visem pesquisar os efeitos da utilização da Acupuntura sobre as respostas fisiológicas ao exercício físico assim como para a melhora do desempenho de atletas (22).

O que se sabe dentro deste contexto que envolve a pesquisa sobre os efeitos da aplicação clinica da Acupuntura sobre o desempenho físico de atletas é que a estimulação de acupontos específicos, tem a capacidade de promover no individuo submetido a sessões de Acupuntura uma maior sensação de bem-estar físico, maior alcance e/ou performance competitiva com conseqüentemente melhora da capacidade de treinamento físico, das qualidades físicas básicas e da plasticidade muscular. Porém conforme relata Luna \& Filho ${ }^{(20)}$, Pelham et al., ${ }^{(19)}$ pouco ainda se conhece sobre os efeitos negativos ou positivos da acupuntura sobre o desempenho físico de atletas.

De acordo com Pelham et al., ${ }^{(19)}$ tais efeitos proporcionadospelaaplicaçãoclinicadaAcupuntura ocorrem pelo fato da Acupuntura atuar sobre o sistema nervoso simpático e parassimpático, sobre o centro da função cardíaca e circulatória o que melhora o desempenho cardiovascular em atletas. E que a técnica de eletroacupuntura produz as mesmas características excitatórias dentro do nervo e/ou músculo que a eletroterapia convencional utilizada pelos fisioterapeutas para aumentar a força e tônus muscular.

Além disso, alguns estudos realizados com outras técnicas de Acupuntura como o desenvolvido por Machado ${ }^{(21)}$, onde foi utilizada a técnica da Crânio Acupuntura de Yamamoto para o tratamento de atletas praticantes de skate com quadro clínico de fratura obliqua de tíbia e fíbula após tratamento traumatológico divididos em dois grupos controle e experimento.

Estudo este que demonstrou que o grupo tratado com a Crânio Acupuntura de Yamamoto apresentou uma redução do quadro álgico em $100 \%$ dos atletas atendidos, redução do tempo de uso do gesso para correção da fratura em $80 \%$ dos atletas, $100 \%$ dos atletas submetidos ao tratamento de Crânio Acupuntura de Yamamoto apresentaram maior taxa de deposição de cálcio superior na região da fratura quando comparados ao grupo controle e ao final do tratamento $100 \%$ dos atletas submetidos ao tratamento de Crânio Acupuntura de Yamamoto relataram não terem observado perda de força muscular no membro inferior afetado quando comparado ao membro inferior não afetado ${ }^{(21)}$.

Ainda no campo de atuação do educador físico os poucos estudos realizados e publicados não se direcionam apenas para estudar, investigar e verificar os efeitos da Acupuntura no rendimento e/ou na recuperação física de atletas, conforme 
descrito anteriormente, mas também tem se direcionado para estudar o conhecimento que os profissionais educadores físicos têm sobre Acupuntura.

Especificamente sobre este assunto localizouse um único estudo realizado por Shinohara ${ }^{(11)}$, na cidade de Castro, estado do Paraná, Brasil, que tinha por objetivo avaliar o conhecimento que profissionais de educação física da cidade tinham sobre a técnica de Acupuntura.

Nesta pesquisa Shinohara (11), observou que $64,9 \%$ dos educadores físicos entrevistados em seu estudo já haviam ouvido falar em Acupuntura, destes $33,33 \%$ desde ouviram falar, mas não sabiam do que se tratava a técnica e $1,8 \%$ nunca tinham ouvido falar em Acupuntura. $96,4 \%$ dos profissionais entrevistados relataram ter conhecimentos sobre algumas patologias e/ ou distúrbios que poderiam ser tratados com a acupuntura. $41,1 \%$ dos profissionais educadores físicos entrevistados por Shinohara ${ }^{(11)}$ referiram saber que a Acupuntura é uma especialidade do educador físico, ao passo que $58,9 \%$ dos profissionais entrevistados referiram não ter tal conhecimento.

Dentre os profissionais que referiram ter o conhecimento de que a Acupuntura é uma especialidade do educador físico $38,8 \%$ destes profissionais afirmaram que qualquer profissional da área de saúde de nível superior e com especializaçãoemAcupuntura incluindo oeducador físico estaria habilitado a praticar acupuntura no Brasil, 33,7\% relataram acreditar que somente o médico especialista em Acupuntura poderia atuar nestaárea, $17,3 \%$ afirmaramacreditarque qualquer profissional de nível superior especializado em Acupuntura estaria habilitado, 5,1\% disseram que qualquer profissional com curso técnico e/ou profissionalizante em acupuntura poderia praticar e nenhum profissional neste estudo afirmou que somente o médico (sem formação especifica em Acupuntura) poderia atuar como acupunturista (11).

Estes dados obtidos no estudo de Shinohara (11) demonstraram que para a maioria dos profissionais de educação física a Acupuntura é uma prática multiprofissional que pode ser exercida por qualquer profissional desde que este tenha formação especifica em Acupuntura. O que torna oportuno neste momento salientar que na China o estudo da Acupuntura é uma questão de dedicação e prática, quanto mais tempo de vivência tem o profissional que lida com Acupuntura, mais valorizado ele é, não importando se este profissional seja um médico, um fisioterapeuta ou um profissional de educação física o que importa é o quanto ele sabe e o quanto ele vivência da Acupuntura.

Muito embora haja quem ainda entenda ser legítima a prática de Acupuntura somente pelo profissional médico. È importante lembrar que todo e qualquer profissional graduado na área da saúde, tem o direito de se especializar e atuar na área de Acupuntura no Brasil conforme a legislação vigente, desde que este siga uma formação em nível superior do tipo graduação /especialização e/ou pós-graduação em Acupuntura e que tenha o exercício profissional da prática de acupuntura devidamente reconhecido pelo seu respectivo conselho. Nestas condições todo e qualquer profissional da área de saúde encontra-se apto a trabalhar com a técnica de Acupuntura dentro do seu respectivo campo de atuação profissional, ou seja, o médico deve utilizar a Acupuntura na área de medicina, o fisioterapeuta na área de fisioterapia e o educador físico na área de educação física e assim por diante. Logo tendo se em vista que o profissional de educação física é um profissional de nível superior e da área de saúde que estuda a anatomia, fisiologia, biologia, psicologia, primeiros socorros, pedagogia, filosofia, cinesiologia, biomecânica, neurofisiologia, psicomotricidade, atividades esportivas, técnicas corporais, mas que sobre tudo é um profissional que estuda o corpo em suas diferentes dimensões. Este profissional encontra-se apto a praticar e atuar na área da Acupuntura desde que possua devida formação nesta área.

Estando ainda este aparado legalmente por uma resolução regulamentadora do seu respectivo conselho desde o ano 2003, que diz que o educador físico pode aplicar os conhecimentos obtidos pela sua formação em Acupuntura no seu respectivo campo de atuação o qual envolve academias de ginástica, clínicas especializadas, spas, hotéis, associações, centros esportivos, resorts, colônias de férias, empresas entre outras áreas que envolvam a cultura física e aos esportes $(23,24)$. Conforme disposto na resolução CONFEF $N^{\circ}$. 069/2003 e no código de ética do educador físico. 


\section{CONSIDERAÇÕES FINAIS}

Mediante o presente estudo pode se concluir que o objetivo principal do estudo foi alcançado, pois se conseguiu abordar os principais aspectos teóricos e políticos que fundamentam a prática de Acupuntura pelo educador físico.

Além disso, foi possível concluir com o presente estudo as seguintes considerações:

- Que existe um consenso sobre o caráter multiprofissional da prática de acupuntura enquanto profissão "especialização" da área de saúde no Brasil por diversos autores;

- Que a Acupuntura é sim uma especialidade multiprofissional na área de saúde reconhecida, aprovada e aparada legalmente por 9 conselhos da área de saúde no Brasil.

- E que desde o ano de 2003 os profissionais de educação física especialistas em Acupuntura podem atuar na área de Acupuntura desde que possuam formação em Acupuntura, que estejam regulamente escritos nos seus respectivos conselhos. Estando assim, portanto estes profissionais aparados legalmente ao exercício de especialidade.

\section{REFERÊNCIAS BIBLIOGRÁFICAS}

1. Matta DF. A educação física no Brasil: Com uma visão transformadora na educação básica, transpirando menos e pensando mais. Revista Latu \& Senso 2001; 2(3):30-3.

2. Verenguer RCG. Intervenção profissional em Educação Física: expertise, credencialismo e autonomia. Revista Motriz 2004; 10(2):123-34.

3. Souza JCA, Bonela LA, Paula AH. A importância do estágio supervisionado na formação do profissional de educação física: uma visão docente e discente. Movimentum Revista Digital de Educação Física 2007; 2(2).

4. Barros LC, Jia JE. Medicina chinesa acupuntura e fitoterapia. São Paulo: Caras S. A; 2004.

5. Conselho Federal de Educação Física. Código de ética do profissional em educação física. Revista ConScientiae Saúde 2004; 3:121-29.
6. Günther MCC, Molina-Neto V. Formação permanente de professores de educação física na rede municipal de ensino de porto alegre: Uma abordagem etnográfica. Revista Paulista de Educação Física 2000; 14(1):85-91.

7. Steinhilber J. Educador Físico poderá aplicar acupuntura. Disponível em: URL: <http://paginas.terra. com.br/saude/jornalnatural/fisico.htm> (10 mai 2009).

8. Almeida-Filho JCA. Regulamentação da profissão de acupunturista ato médico ou corporativismo?. [Tese]. Rio de Janeiro: UGF; 2003. p.49.

9. Akiyama K. Práticas não-convencionais em medicina no município de São Paulo. [Tese]. São Paulo: USP; 2004. p.105.

10. Pai HJ. Acupuntura de terapia alternativa a especialidade médica. São Paulo: Ceimec; 2005.

11. Shinohara ES. Mapeamento do conhecimento da acupuntura no âmbito dos profissionais de educação física na cidade de Castro - PR. [Monografia]. Castro: FAEFIJA/IBRATE; 2007. p.96.

12. ZambotiAS. Percepção e conhecimento do acadêmico de enfermagem frente a pratica da acupuntura por enfermeiros - Estudo de caso. [Monografia]. Cascavel: FAEFIJA/IBRATE; 2007. p.109.

13. Nöthlich. Afinal, quem pode praticar a Acupuntura no Brasil?. Disponível em: URL: <http://acupuntura.pro.br/ legislacao/quem-pode-praticar/.> (15 mai 2009).

14. Silva JAÁ. Portaria n. 971, de 3 de maio de 2006. Diário Oficial da União poder Executivo, Brasília, Seção 1, p. 20-25. 2008.

15. Hoshino MS. Fisioterapeuta Acupunturista: Atuação profissional e expectativa de trabalho. [Monografia] Cascavel: Universidade Estadual do Oeste do Paraná; 2004. p.63.

16. Wen TS. Acupuntura Clássica Chinesa. $14^{\mathrm{a}} \mathrm{ed}$. rev. São Paulo: Cultrix; 2009.

17. Maike SRL. Fundamentos essenciais da acupuntura chinesa. São Paulo: Ícone; 1995.

18. Nozabieli AJL, Fregonesi CEPT, Fregonesi DA. Correlação dos canais de acupuntura com a neuroanatomia e a neurofisiologia. Arquivos de Ciências da Saúde da Unipar 2002; 4(3):263-68. 
19. Pelham TW, Holt LE, Stalker R. Acupuncture in human performance. The journal of strength and conditioning research 2001; 5(2):266-71.

20. Luna MP, Filho JFF. Efeitos da Acupuntura na Performance de Atletas Velocistas de Alto Rendimento do Rio de Janeiro. Fitness \& Performance Journal 2005; $4(4): 1-32$.

21. Machado CMP. Os efeitos da Cranioacupuntura de Yamamoto como recurso terapêutico em atletas praticantes de skate com quadro de fratura oblíqua de tíbia e fíbula. [Monografia]. Curitiba: FAEFIJA/IBRATE; 2007. p.57.

22. Rosetto SC, Callado JC. O uso da acupuntura para a melhora de performance física em atletas. I Congresso de Fisioterapeutas Acupunturistas, 2003, Uberlândia. Anais do I Congresso - SOBRAFISA, 2003. v.1.
23. Pimentel GGA. Educação física e atuação profissional no lazer. Revista eletrônica "Novas Atenas" de educação tecnológica, 2002; 5(1):1-12. Educação física e atuação profissional no lazer. Disponível em: URL: < www.crefsc. org.br/artigos/acupuntura.doc.> (15 mai 2009).

24. Verenguer RCG. Mercado de trabalho em educação física: reestruturação produtiva, relações de trabalho e intervenção profissional. Revista Mackenzie de Educação Física e Esporte 2005; 4(4):39-54.

Endereço para correspondência:

ANA PAULA SERRA DE ARAÚJO, Rua Natal, nº, 2982, CEP: 87504-230, Centro, Umuarama, Paraná, Brasil. Tel: (44)3624-2003/(44)9129-6105. E-mail: anaps_ araujo@hotmail.com / anasaraujo@hotmail.com 